\title{
A dual threshold listen-before-talk for unlicensed LTE systems
}

\author{
Jae-Hoon Kim ${ }^{1 *}$ and Sungchul Choi ${ }^{2}$
}

\begin{abstract}
The insufficient wireless frequency bands are likely to arise with the increase in mobile traffic demand. Many mobile operators suffer from a high annual increase in the cost of licensed wireless bands. Unlicensed bands present a potential opportunity for mobile operators. A single 5-GHz band with a large bandwidth provides faster transmission for each user. However, interference caused by existing wireless transmissions, such as Wi-Fi in unlicensed bands, is a serious issue in the effective utilization of unlicensed bands by mobile operators. The Listen-Before-Talk (LBT) was proposed as a promising method to ensure the coexistence of Wi-Fi and commercial mobile services such as LTE. We focus on effective control of the contention window (CW) size to maximize the operability of LBT. Control of the CW size depends on the repetition of extended clear channel assessment (ECCA). We introduce a dual threshold system that counts the repetition of ECCA. The newly developed NS-3-based unlicensed band network simulator shows the effectiveness of the proposed control mechanism. The results indicated that the advantage of the proposed method improves $4.3-7.6 \%$ than traditional LBT method. We develop a composite metric of throughput and fairness to evaluate the performance of LTE-LAA access.
\end{abstract}

Keywords: LTE, Wi-Fi, Coexistence, ns-3, ECCA, Simulation, LBT, Unlicensed, 5 GHz

\section{Introduction}

The widespread use of mobile devices and various highquality contents cause a significant increase in mobile internet traffic. VR/AR media and personal video streaming, such as Facebook Live, cause an exponential increase in traffic. A report by Cisco indicates that mobile connections will reach 11.5 billion and global mobile data traffic will exceed 24.3 exabytes in 2020 [1]. The explosion of mobile data traffic increases the need of additional wireless bandwidth. Although licensed wireless bands are extremely affordable with respect to guaranteeing service quality, the usability of licensed bands is strictly limited. Limited amounts of licensed bands (i.e., $20-40 \mathrm{MHz}$ for single-LTE operators) are allocated to mobile operators under strict regulations by domestic governments. Specifically, LTE Licensed-Assisted Access (LTE-LAA) proposes aggregated carrier utilization between licensed and unlicensed bands (see Fig. 1). A large unlicensed bandwidth (i.e., $455 \mathrm{MHz}$ bandwidth in Europe and $580 \mathrm{MHz}$ in the USA in the 5-

\footnotetext{
* Correspondence: jayhoon.kim@gmail.com

${ }^{1}$ Department of Industrial Engineering, Ajou University, 206, World cup-ro,

Yeongtong-gu, Suwon-si, Gyeonggi-do 16499, Korea

Full list of author information is available at the end of the article
}

$\mathrm{GHz}$ band) can be used as sufficient supplementary carriers under the assistance of control signaling of licensed bands.

Current unlicensed bands are widely used for various wireless access systems. Wi-Fi systems, such as IEEE $802.11 \mathrm{ac}$, present an access method over the unlicensed bands [3]. Fair radio resource sharing between Wi-Fi access systems and LTE-LAA is a key issue in the utilization of unlicensed bands. A traditional LTE system promptly transmits signals without censoring the channel condition (i.e., static resource scheduling is applied to LTE systems), and thus, it is not possible to simply share radio resources with Wi-Fi wireless access systems. Therefore, a novel technical standard for the LTE-LAA channel access method is required to guarantee fair radio resource sharing with $\mathrm{Wi}-\mathrm{Fi}$ access systems over unlicensed bands. A study by Dimatteo et al. proposed an integrated architecture to exploit the opportunistic networking paradigm. They built a data traffic migration method from cellular networks to metropolitan Wi-Fi access points [4]. Jeon et al. focused on the mutual effect between LTE and Wi-Fi systems. They developed an intersystem interference analysis technique based on continuum field approximation and spiral representation [5]. Liu et al. proposed a simultaneous access framework for 


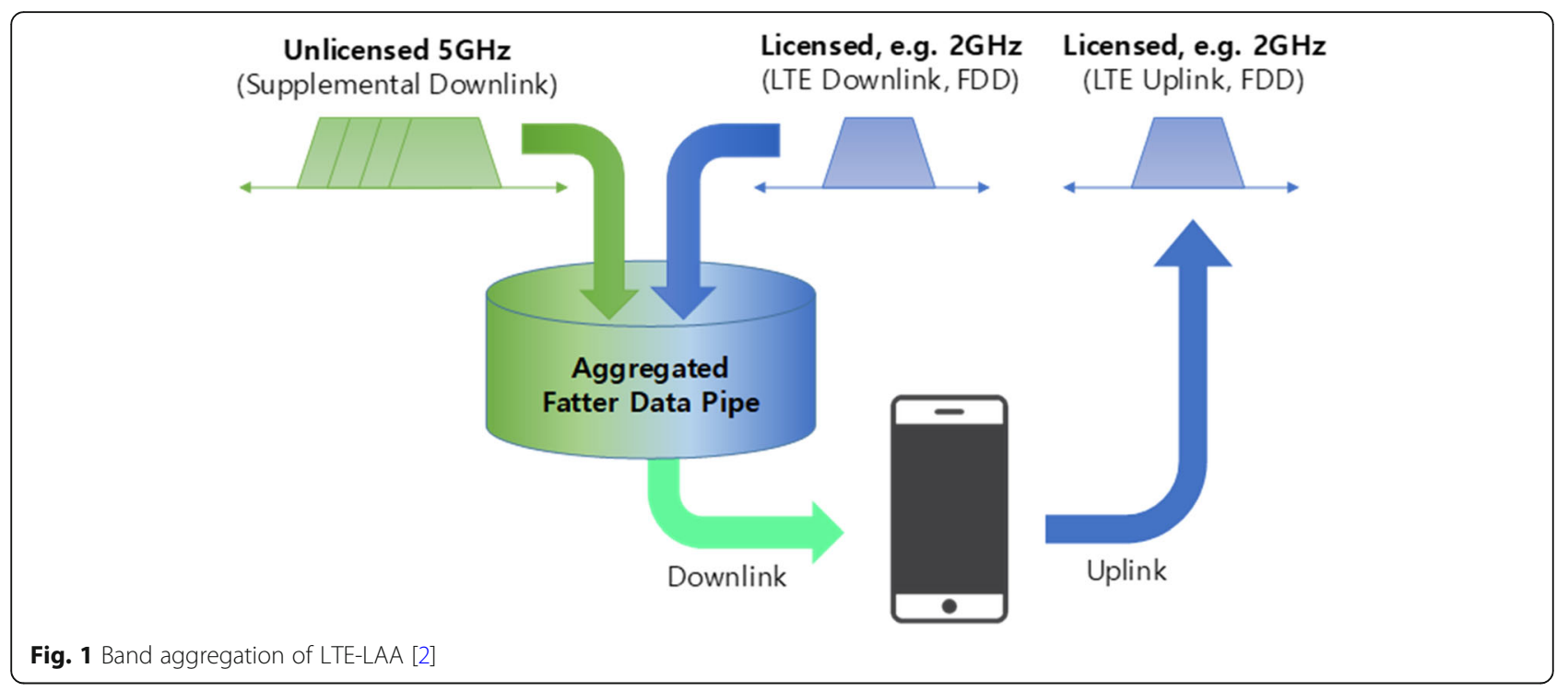

small cells over licensed and unlicensed bands. A small cell dynamically arranges its channel access system parameters for effective coexistence with incumbent unlicensed band users. The coexistence was analytically modeled and proved via extensive simulations [6]. Cavalcante et al. compared performance evaluations of coexistence between LTE and Wi-Fi systems and pointed out technical challenges with respect to the two different access systems. They focused on a system-level analysis for specific environments such as an office or small closed area. The simulation results indicated that the LTE system performance is slightly affected by coexistence, while Wi-Fi is significantly affected by LTE transmissions [7]. A study by Almeida et al. suggested a simple coexistence scheme that reused the concept of almost blank subframes (ABS) in LTE [8]. The use of ABS is an essential point of LTE unlicensed $[9,10]$. Major wireless technology companies, such as Qualcomm or Ericsson, have focused on Carrier Sensing Access Technology (CSAT) for LTE adaptation over unlicensed bands.

The Listen-Before-Talk (LBT) framework is a de facto standard of LTE-LAA channel access methods to aid in fair sharing over unlicensed bands. The adjusting LTE MAC protocols for adopting LBT are proposed [11]. It concludes that LTE-LAA can gain high-throughput performance without harming Wi-Fi performance with the proposed MAC mechanisms. The study of Ratasuk et al. [12] proposed a fairness mechanism for LAA coexistence. Downlink system performance is analyzed for multioperator LAA deployment in an indoor hotspot, an indoor office, and an outdoor small cell. LBT similar to Wi-Fi DCF presented good fairness with Wi-Fi. But, they only hold when the coexistence channel model can accurately simulate the interference. An analysis by Chen et al. proved the effective sharing of LTE-LAA and Wi-Fi over the downlink unlicensed bands [13]. They applied a
Markov chain model to a simple LBT method. Um et al. investigated the effectiveness of two alternatives, framebased equipment (FBE) and load-based equipment (LBE), as practical implementations for LBT. The results indicated that LBE has an advantage in terms of flexible channel waiting time on busy periods despite the complexity of the LBE implementation [14, 15]. The extensive survey of LBT is provided in the study of Chen et al. [16]. They provided a holistic study for not only coexistence schemes but also overall operations of LBT.

The backoff time defined in LTE-LAA plays an important role in how the traffic will be split between Wi-Fi and LTELAA and, hence, is a factor in how fair the coexistence will be [17]. The adoption of an extended clear channel assessment (ECCA) for LBE granted flexibility with respect to the design of a backoff time control algorithm. When a channel is subject to a highly competitive condition, sufficient backoff time can be achieved by a sufficient contention window (CW) while minimizing unnecessary waiting time. In the study of 3GPP [18], they proposed the design of three LBT design options: (1) LBT without random backoff, (2) LBT with random backoff in a contention window of fixed size, and (3) LBT with random backoff in a contention window of variable size. Yin et al. [19] presented the minimum CW size adaptively adjusted according to the available licensed spectrum. An analytical model was developed to estimate the collision probability. Tao et al. [20] focus on CW size adaptively adjusted to an appropriate value by means of collecting the QoS metric signals from neighbor mobile stations. They assumed that the information can be exchanged over the inter-cell interfaces such as X2 interface. Fanrong et al. [21] determined the $\mathrm{CW}$ size based on the slot utilization. They built the analytical model of LTE-LAA slot structure and calculated slot utilization rate. Baswade and Tamma [22] also suggested CW size adjustment based on 
waiting time that can be directly derived from slot utilization. Saadat et al. [23] suggested a variety of CW size control method. They presented LAA-Wi-Fi coexisting scheme with transmission opportunity (TXOP) backoff control method. Instead of doubling backoff time for every unsuccessful transmission attempt, TXOP is reduced by half. TS37.213 (rel. 15) [24] presents the current LBT implementation of 3GPP LTE-LAA. The basic structure of 3GPP implementation is similar to LBE or FBE. It employs the $\mathrm{CW}$ and a counter. The 3GPP makes a categorization by the channel access priority. The higher access priority has smaller CW size. The smaller CW can guarantee the more opportunity to access channels.

The aforementioned research works have their own importance and try to make meaningful insights. However, they have some limitations to describe the practical LTELAA operations. First, almost all CW update procedures are based on LAA user feedback and base station assessment on a relatively slow time scale. They need the estimation of available licensed bandwidth and load over the Wi-Fi network. In addition, they assume the adoption of inter-cell interfaces such as $\mathrm{X} 2$ interface for the information exchange. The frequent measurements from the Wi-Fi networks and information exchange via separated interfaces are actually unrealistic. The hardware and software limitation of LTE-LAA networks (especially for small cell LTE-LAA networks) restricts the sufficient inter RAT (Radio Access Technology) measurements and separated physical interfaces between networks. Moreover, the analytical channel access model such as a Markovian model considered in the aforementioned works has only a limited description for contention stage. The environmental variations over network are not sufficiently considered.

In the present study, an effective design for the CW control algorithm of LBT is proposed. The proposed design suggests a dual threshold structure with respect to the CW control method to prevent improper ascending or descending relative to the waiting time. Extensive simulations and intuitive gradient-descending method indicate that the proposed dual threshold structure achieved high performance while guaranteeing fair radio resource sharing with Wi-Fi systems. We need only simple counting on unsuccessful transmission trials. The complicated analytical model and information exchange between networks are not required. Note that, we assume that all mobile nodes have same access priority to estimate holistic performance of access technologies. The study is organized as follows: A dual threshold design under the ECCA structure is suggested in Section 2. The gradient control of the dual threshold is proposed in Section 3. The computational results are analyzed in Section 4. The concluding remarks are presented in Section 5 .

\section{ECCA structure and channel access method}

\subsection{Background}

The ECCA-based channel access method is based on the European standard LBE [12]. A mobile device performs a clear channel assessment (CCA) using "energy detect" prior to a transmission over an operating channel. A mobile device performs observations over the operating channel with respect to the CCA sensing time (that typically corresponds to less than $18 \mu \mathrm{s})$. The operating channel is considered as occupied if the detected energy level over the operating channel exceeds the predefined level. The mobile device commences ECCA sensing when the mobile device detects channel occupation within the CCA sensing time. The ECCA sensing time is randomly selected within (CCA Sensing Time $\times(1, \cdots, q)$ ), where $q$ denotes the maximum ECCA sensing time. The mobile device may resume transmissions (i.e., TXOP state, less than $10 \mathrm{~ms}$ ) over the operating channel if the ECCA sensing identifies that the operating channel is not occupied. Following the ending of the TXOP state, the mobile device continues a short control signal transmission time (usually equal to CCA sensing time) over the operating channel, and this provides management and control frames (e.g., ACK and Block ACK frames). The LBE estimates channel contention by measuring consecutive repetitions of ECCA sensing. A new ECCA is repeatedly activated whenever the operating channel is occupied by other devices. The state transition diagram shown in Fig. 2 represents the details of the ECCA structure.

\subsection{Proposed access control}

The ECCA sensing time is denoted by a function of $q$ (i.e., $C C A$ Sensing Time $\times(1, \cdots, q))$. We introduce two counts to determine an effective $q$ value, namely an ECCA increase counter and an ECCA decrease counter. The ECCA increase counter is used to determine the increasing event of ECCA sensing time. When the ECCA increase counter reaches $i T H$ (i.e., ECCA increase threshold), the $q$ value is doubled and the ECCA sensing time can then be increased. The $E C C A$ decrease counter is used to determine the decreasing event of the ECCA sensing time. When a mobile device is in a high contention environment, the $E C C A$ increase counter by itself results in an unnecessarily long waiting time to gain access over the operation channel. The ECCA decrease counter and $d T H$ (i.e., ECCA decrease threshold) can guarantee the minimum accessibility of a device in a high contention environment. The ECCA increase counter simply increases at the beginning of each ECCA. Whenever ECCA increase counter equals $i T H$, the $q$ value is doubled and the $E C C A$ increase counter resets to 0 . The ECCA decrease counter also increases at the beginning of each ECCA, but it retains the same value when the $q$ value is doubled. If the $E C C A$ decrease counter reaches $d T H$, then the $q$ value resets to the minimum and ECCA decrease counter resets to 0 . The $E C C A$ decrease counter is increased by 1 only when 


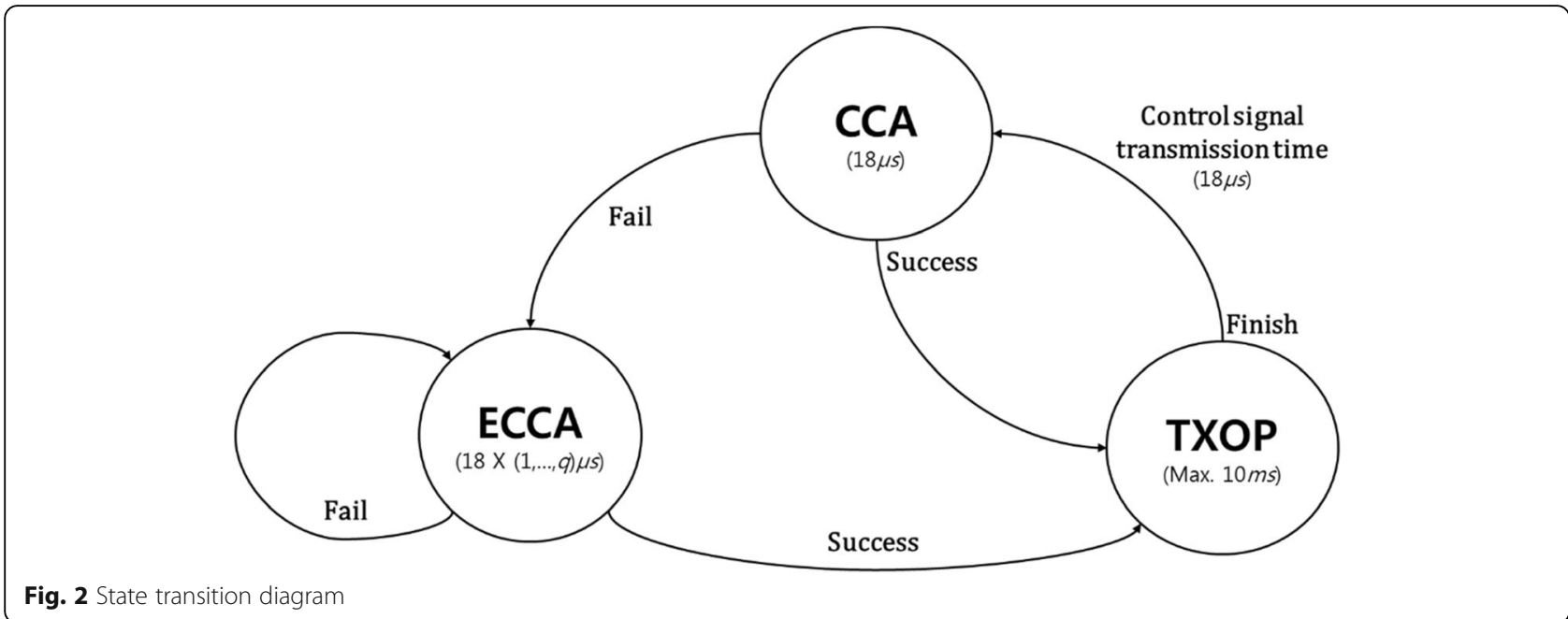

the value of the $E C C A$ increase counter is smaller than $i T H$. Thus, ECCA sensing time only decreases when the ECCA decrease counter corresponds to $d T H$. These dual counters and thresholds allow a simple but effective method of LBT implementation. Figure 3 shows the access control process flow chart. Figure 3a represents an overall access control process in which ECCA sensing time calculation is included as a submodule. Figure $3 \mathrm{~b}$ provides a detailed calculation procedure for the ECCA sensing time.

The maximum ECCA sensing time is determined by the $q$ value. The maximum ECCA sensing time doubles when the ECCA increase counter reaches $i T H$. In contrast, the maximum ECCA sensing time resets to the minimum value when the value of the $E C C A$ decrease counter corresponds to $d T H$. Figure 4 illustrates examples of operational actions of counters and thresholds. The success of channel access is strongly dependent on the ECCA sensing time. An increase in the ECCA sensing time results in a lower success rate of the LTE channel access. The proposed dual threshold method presents a mixing strategy of the short and long ECCA sensing times even over an overloaded channel. As shown in Fig. 4, the proposed dual threshold method guarantees that the LTE retains channel occupancy while permitting sufficient channel access chance to Wi-Fi devices. It is assumed that $i T H$ and $d T H$ correspond to 4 (i.e., $i T H=d T H=4$ ), and Wi-Fi transmission is occasional. The \#/\# form values denote $\{E C C A$ increase counter/ECCA decrease counter\} for each ECCA beginning. The red dotted rectangle depicts that $E C C A$ increase counter reaches $i T H$. When the ECCA increase counter reaches $i T H$, the value of the $E C C A$ increase counter immediately drops from 4 to 0 ( $E C C A$ decrease counter continues to remain at 3 ), and the $q$ value is doubled. The blue dotted rectangle shows the actions of the ECCA decrease counter. Whenever the value of the ECCA decrease counter reaches $d T H$, the value of the $E C C A$ decrease counter decreases from 4 to 0 , and the $q$ value decreases to the minimum value. The operation of the ECCA decrease counter allows more frequent access trials for the LTE over the overloaded channel.

\section{Gradient control of dual threshold}

The two thresholds (i.e., $i T H$ and $d T H$ ) induce a critical impact on the LBT performance over the operating channel. The ECCA increase threshold, $i T H$, denotes the consecutive occurrence of ECCA that is required to double the maximum ECCA sensing time (i.e., $q$ value). Higher values of $i T H$ restrict the increase in the $q$ value, and the ECCA sensing time is then slowly extended. An increase in the ECCA sensing time results in fewer Wi-Fi access trials. Lower $i T H$ values cause a frequent increase in the $q$ value. A rapid increase in the $q$ value results in excessive ECCA sensing time that reduces the access trials of LTE. The behavior of $d T H$ can be interpreted in a similar manner. Higher values of $d T H$ restrict the possibility of initializing the $q$ value, and subsequently, the channel access of LTE is excessively limited. Lower $d T H$ values result in the frequent initialization of the $q$ value. The excessively short ECCA sensing time poses a serious disadvantage for Wi-Fi access trials. Performance metrics are typically designed as a combining function of throughput and fairness for the two given thresholds. The throughput is easily obtained by a summation of the traffic throughputs of the LTE and Wi-Fi while the fairness is indirectly estimated by the weighted difference between the traffic throughputs of the LTE and Wi-Fi.

$$
\begin{aligned}
& \text { throughput }(i T H, d T H) \\
& \quad=\sum_{i} r_{i}^{L T E}+\sum_{j} r_{j}^{W i-F i} \\
& \text { fairness }(i T H, d T H)=w \times\left|\sum_{i} r_{i}^{L T E}-\sum_{j} r_{j}^{W i-F i}\right|
\end{aligned}
$$

where $r_{i}^{L T E}$ denotes the $i$ th traffic throughput of LTE flow and $r_{j}^{W i-F i}$ denotes the $j$ th traffic throughput of Wi-Fi flow. 


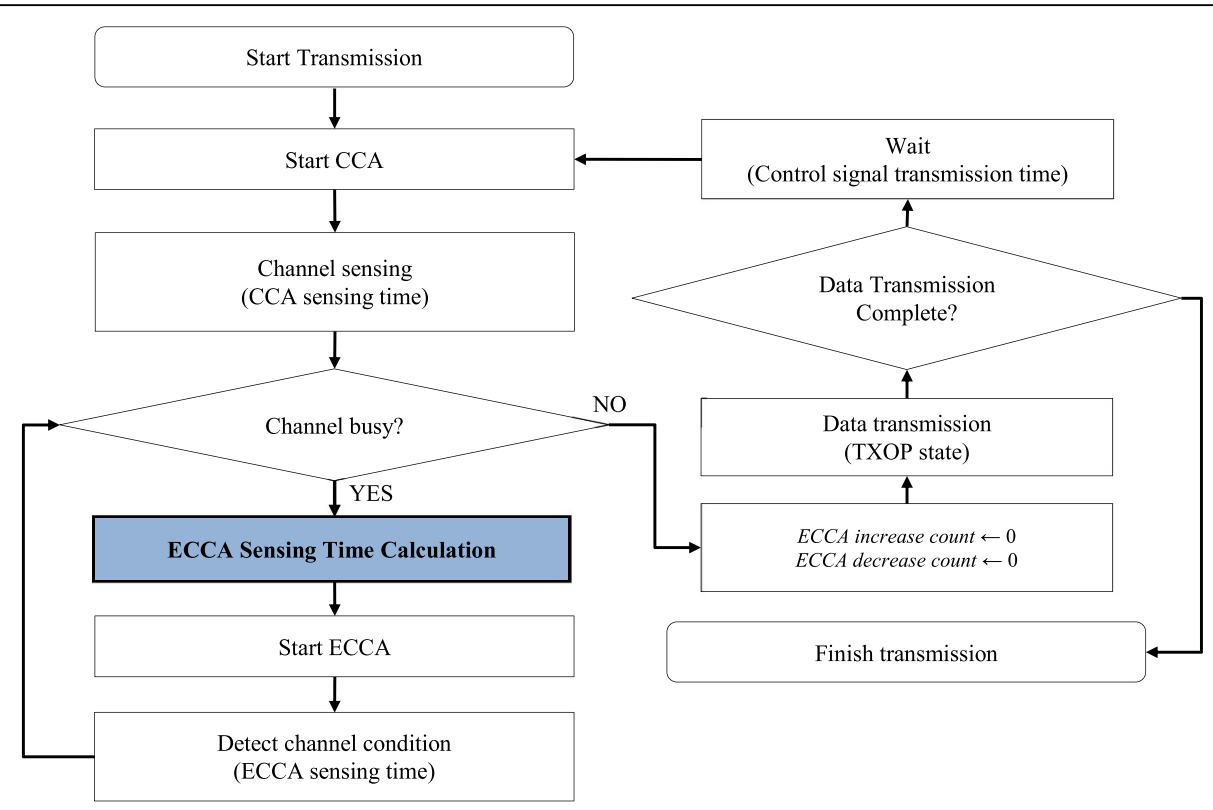

(a) Overall Access Control Process

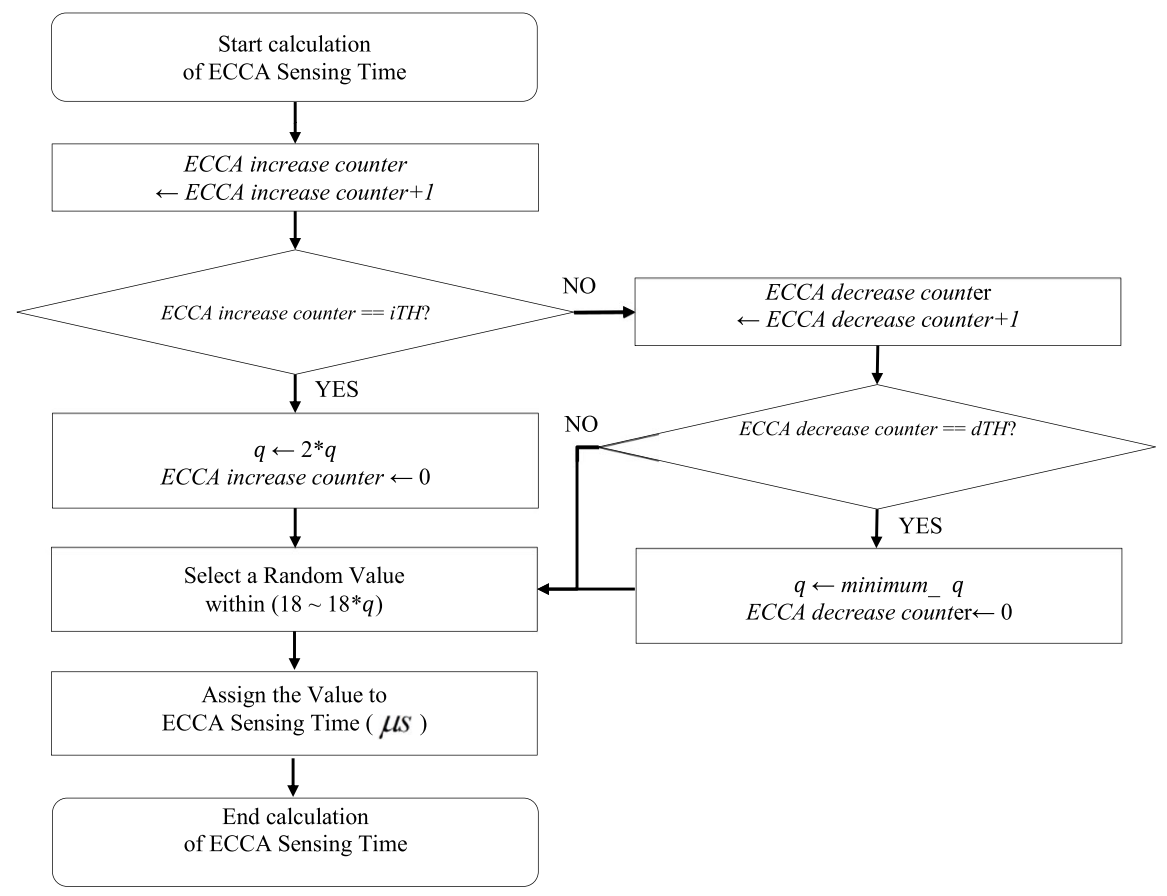

(b) ECCA Sensing Time Calculation

Fig. 3 Flow chart for access Control. a Overall access control process. b ECCA sensing time calculation

Additionally, $w$ denotes the applied weight of the throughput difference. The theoretical bases of LTE unlicensed band and Wi-Fi $5 \mathrm{GHz}$ are similar. They both use OFDM and LBT technology. The performance difference is only originated from the bandwidth allocation. The allocated bandwidth is directly related to its throughput. The throughput is essential resource to users. The users share the total system throughput. The fair bandwidth allocation guarantees the fair system throughput between two technologies where two user groups of two technologies can share the throughput. Therefore, the throughput difference between two technologies directly presents the fairness between two technologies.

Note that the throughput (1) and fairness (2) calculation are performed by simulations. We build a simulation 


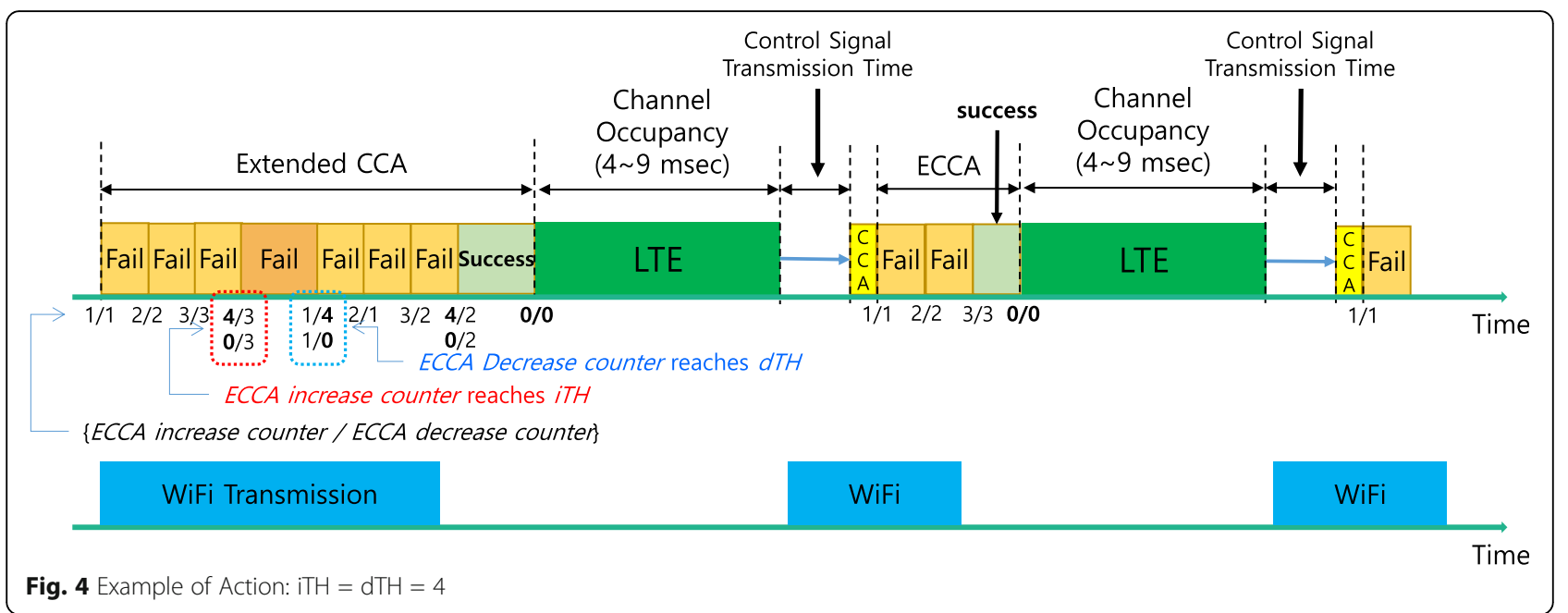

environment and then extract representative simulation results to calculate throughput and fairness (Table 3 in the Appendix shows the abstracted simulation results).

Both the throughput and fairness functions have two independent variables of $i T H$ and $d T H$. Figure 5 shows an example of the LTE throughput and Wi-Fi throughput. The $x$ axis and $y$-axis denote $i T H$ and $d T H$, respectively, and the $z$-axis denotes the throughput (in Mbps). The system setup is described in the following Section 4.1. The throughput function (1) is directly illustrated as the uppermost gray surface in Fig. 5 . The fairness function (2) can be estimated by the weighted difference between LTE throughput (denoted by the light red surface) and Wi-Fi throughput (denoted by the light blue surface). The value of the fairness function is close to zero when the throughput difference between the LTE and Wi-Fi converges to a minimum value.

The determination of proper $i T H$ and $d T H$ values is an essential part of the proposed CW control method to maintain a balance between LTE and Wi-Fi. Function (3) shows a functional model to obtain proper values of $i T H$ and $d T H$ as follows:

$$
\begin{aligned}
\arg _{(i T H, d T H)} \max f(i T H, d T H) & =\{(i T H, d T H) \mid f(i T H, d T H) \\
& =\text { throughput }(i T H, d T H) \\
& \text {-fairness }(i T H, d T H)\}
\end{aligned}
$$

It is hard to model the $f(i T H, d T H)$ as a closed form due to the irregular discreteness of $f(i T H, d T H)$. However, the functional form can be approximated by behavioral pattern analysis for the dual threshold $(i T H, d T H)$. Figure 5 shows the typical behaviors of LTE and Wi-Fi throughputs. The Wi-Fi throughput linearly increases with respect to $d T H$ and exponentially decreases with respect to $i T H$. The LTE throughput logarithmically increases relative to $i T H$ and exponentially decreases relative to $d T H$ as follows:

$$
\begin{aligned}
& \text { Wi-Fi throughput }=\theta_{1} d T H+\theta_{2} e^{i T H}+\theta_{3} \\
& \text { LTE throughput }=\theta_{4} \log (i T H)+\theta_{5} e^{d T H}+\theta_{6} \\
& f(i T H, d T H)=\left(\theta_{1} d T H+\theta_{2} e^{i T H}+\theta_{3}+\theta_{4} \log (i T H)+\theta_{5} e^{d T H}+\theta_{6}\right) \\
& -w\left(\theta_{1} d T H+\theta_{2} e^{i T H}+\theta_{3}-\theta_{4} \log (i T H)-\theta_{5} e^{d T H}-\theta_{6}\right) \\
& =(1-w)\left(\theta_{1} d T H+\theta_{2} e^{i T H}+\theta_{4} \log (i T H)+\theta_{5} e^{d T H}+\theta_{3}+\theta_{6}\right)
\end{aligned}
$$

To determine proper $(i T H, d T H)$, the parameters $\theta_{i}(i=1$, $\cdots, 6)$ should be estimated appropriately. The gradient decent method [25] is very useful for multivariate function parameter estimation. The function, $f(i T H, d T H)$, has a good presentation to apply the gradient decent method (Fig. 5 shows a typical decent direction to optimize the function $f(i T H, d T H))$. The selected results of LTE and Wi-Fi throughput are already gathered by the carefully designed network simulator (see Table 3 in appendix). For efficient application of the gradient decent method, we slightly modify the function $f(i T H, d T H)$ (Eq. (6)) as follows (Eq. (7)).

$$
h_{\xi}(x)=\xi_{0}+\xi_{1} x_{1}+\xi_{2} x_{2}+\xi_{3} x_{3}+\xi_{4} x_{4}
$$

where $x_{1}=d T H, x_{2}=e^{i T H}, x_{3}=\log (i T H), x_{4}=e^{d T H}, \xi_{0}=(1$ $-w)\left(\theta_{3}+\theta_{6}\right), \xi_{1}=(1-w) \theta_{1}, \cdots, \xi_{4}=(1-w) \theta_{4}$.

The cost function, $J\left(\xi_{0}, \cdots, \xi_{4}\right)$, which present the deference between functionally estimated and actually simulated performances, is given as Eq. (8).

$$
J\left(\xi_{0}, \cdots, \xi_{4}\right)=\frac{1}{2 m} \sum_{i=1}^{m}\left(h_{\xi}\left(x^{(i)}\right)-y^{(i)}\right)^{2}
$$

where $x^{(i)}=\left(d T H, e^{i T H}, \log (i T H), e^{d T H}\right)^{(i)} \quad$ (i.e., variables from the $i$ th dual thresholds $(i T H, d T H)$ ) and $m$ is the number of simulated performances in Table 3 in Appendix.

The value, $y^{(i)}$, is obtained from the $i$ th simulated performance ofthroughput $(i T H, d T H)$ - fairness $(i T H, d T H)$ 


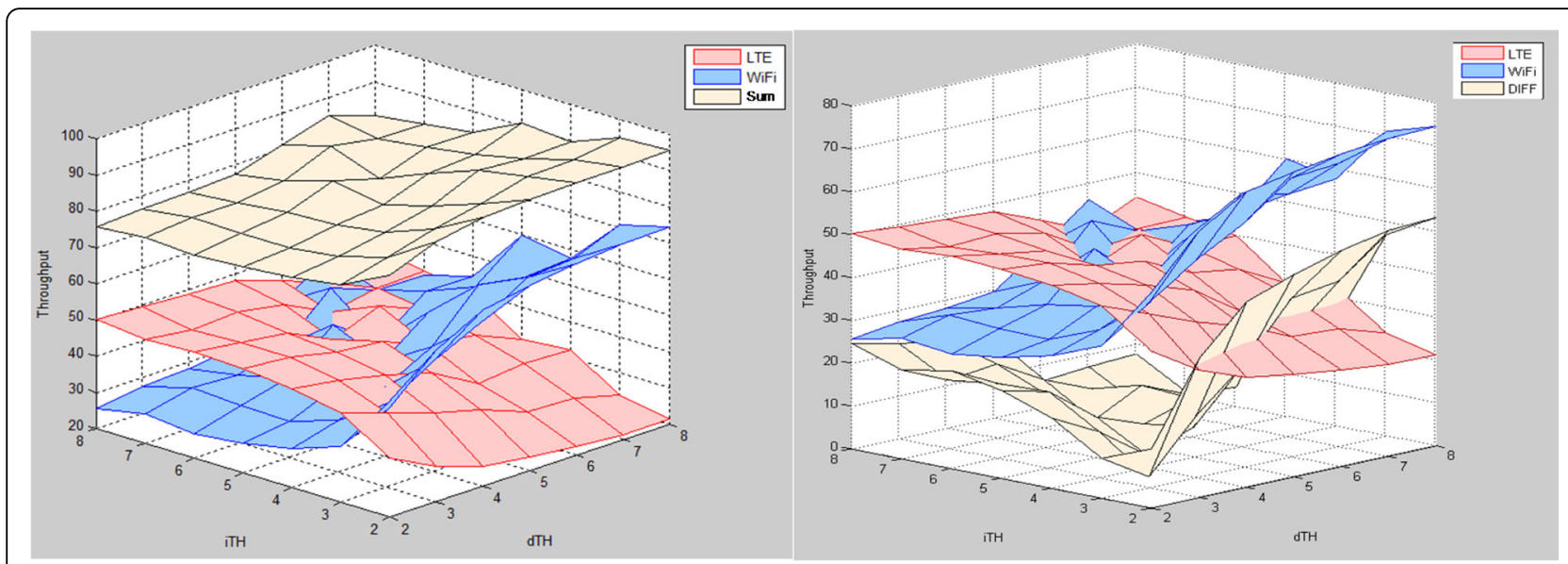

(a) Throughput Summation

(b) Fairness

Fig. 5 3D Throughput analysis. a Throughput summation. b Fairness

(see Table 3 in Appendix). The gradient decent for parameter $\xi_{j}$ 's are described as following (9).

$$
\xi_{j}:=\xi_{j}-\alpha \frac{1}{m} \sum_{i=1}^{m}\left(h_{\xi}\left(x^{(i)}\right)-y^{(i)}\right) x_{j}^{(i)} \text { for } j=\{0, \cdots, 4\}
$$

where $\alpha$ is the learning rate (i.e., the smaller $\alpha$ denotes slower learning and vice versa).

The training data set $\left(x^{(i)}, y^{(i)}\right)$ determines the proper parameters of function (7). Table 3 in the Appendix shows the examples of training data set. The input instances, $x^{(i)}$, are obtained from $(i T H, d T H)$, and output values, $y^{(i)}$, can be obtained from the small number of simulations. After determining the parameters, $\xi_{0}, \cdots \xi_{4}$, we can estimate the holistic behavior of function (6) for every dual threshold $(i T H$, $d T H$ ) (i.e., we calculate $\theta_{1}, \cdots, \theta_{6}$ of function (6) using $\xi_{0}$, $\left.\cdots \xi_{4}\right)$. However, the nonlinearity of function (6) given as $(i T H, d T H)$ cannot guarantee a proper finding of dual threshold. A search method is applied as follows:

$$
\left(i T H^{k+1}, d T H^{k+1}\right)=\left(i T H^{k}, d T H^{k}\right)+\delta^{k} \nabla f\left(i T H^{k}, d T H^{k}\right)
$$

$$
\nabla f\left(i T H^{k}, d T H^{k}\right)=\left\{\begin{array}{cl}
(-1,0) & \text { if } f\left(i T H^{k}-1, d T H^{k}\right)-f\left(i T H^{k}, d T H^{k}\right)>0 \\
(+1,0) & \text { if } f\left(i T H^{k}+1, d T H^{k}\right)-f\left(i T H^{k}, d T H^{k}\right)>0 \\
(0,-1) & \text { if } f\left(i T H^{k}, d T H^{k}-1\right)-f\left(i T H^{k}, d T H^{k}\right)>0 \\
(0,+1) & \text { if } f\left(i T H^{k}, d T H^{k}+1\right)-f\left(i T H^{k}, d T H^{k}\right)>0 \\
(0,0) & \text { otherwise }
\end{array}\right.
$$

It is necessary to update the $k$ th dual threshold, $\left(i T H^{k}\right.$, $d T H^{k}$ ), by the increasing direction of function $f$. The increasing direction, $\nabla f$, has a unit vector form (i.e., $( \pm 1,0)$ or $(0, \pm 1))$. The difference in the function values between $k$ th dual threshold and its one step neighbors (i.e., $f\left(i T H^{k} \pm 1\right.$, $\left.d T H^{k}\right)-f\left(i T H^{k}, d T H^{k}\right)$ or $f\left(i T H^{k}, d T H^{k} \pm 1\right)-f\left(i T H^{k}, d T H^{k}\right)$

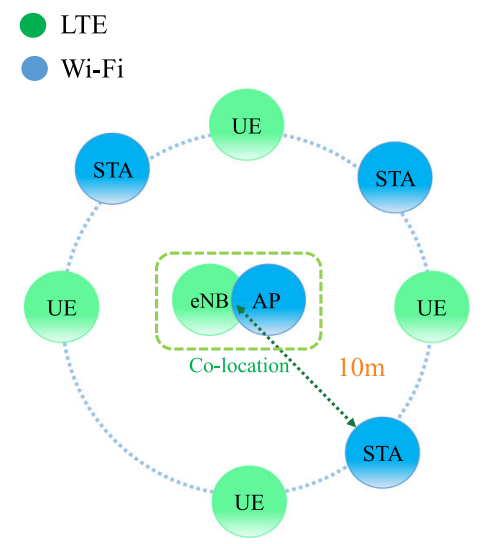

(a) Low Interference Environment

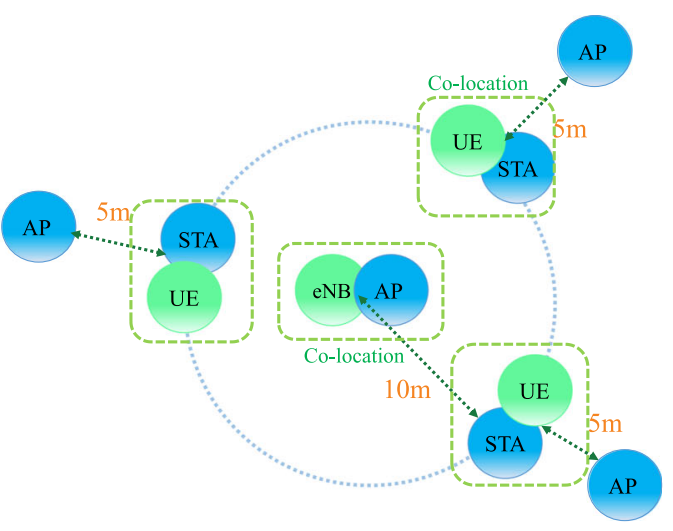

(b) High Interference Environment

Fig. 6 Simulation environments. a Low interference environment. b High interference environment 
determines the increasing direction, $\nabla f$. Additionally, $\delta^{k}$ denotes a step size for the $k$ th dual threshold. The $k+1$ th dual threshold $\left(i T H^{k+1}, d T H^{k+1}\right)$ is determined by the product of the $k$ th increasing direction and the step size. The operation commences with a wide step size for fast improvement, and the step size is then gradually reduced for minor calibration. The dual threshold update ends without any significant improvement with respect to function $f(i T H, d T H)$. If $f\left(i T H^{k+1}, d T H^{k+1}\right)-f\left(i T H^{k}, d T H^{k}\right)<\varepsilon$, then the threshold update is stopped and the current dual threshold, $\left(i T H^{k}, d T H^{k}\right)$, is reported as the best threshold.

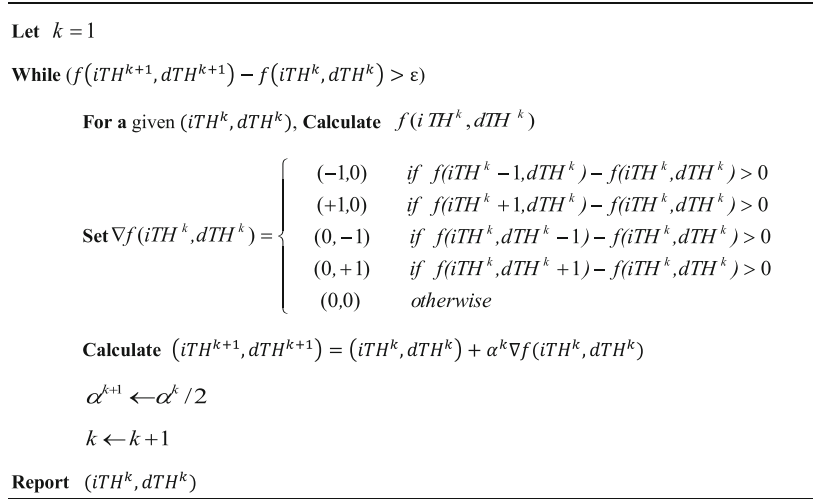

Note that, the solution processes of optimization problem are derived by an offline application. The active code of proposed gradient decent method is very simple to activate in small cell eNB. We check the capability of commercial eNB. The installed CPU of small cell eNB is Intel SoC T3300(2Core, $2 \mathrm{GHz}$ ). The computing overhead of proposed gradient descent method is less than $1 \%$ of T3300.

\section{Computational results}

\subsection{A system model for simulations}

The test analysis was performed using NS-3, which is a $\mathrm{C}++-$ based discrete event simulator. Both LTE and Wi-Fi operate over $5180 \mathrm{MHz}$ of center frequency with a bandwidth of $20 \mathrm{MHz}$. The transport protocol is dedicated to UDP for pure data rate measurement. The Wi-Fi operation is dictated by IEEE $802.11 \mathrm{n}$. The CCA time is assigned as $18 \mu$ s to implement the proposed dual threshold method. The $q$ value has a variable range corresponding to 5-30. Additionally, the single-channel occupation time (i.e., TXOP time) for the transmission is assigned as $8 \mathrm{~ms}$. The simulation time is set to $30 \mathrm{~s}$, and the analysis is performed for the following environments (as shown in Fig. 6).

Two interference environments are constructed for test cases. Figure 6a shows a low-interference environment in which 1 eNodeB and $1 \mathrm{Wi}-\mathrm{Fi}$ AP are co-located at the
Table 1 Basic LTE/WI-FI system model

\begin{tabular}{|c|c|c|c|}
\hline \multicolumn{3}{|l|}{ Parameters } & Values \\
\hline \multicolumn{3}{|l|}{ Center frequency } & $5180 \mathrm{MHz}$ \\
\hline \multicolumn{3}{|l|}{ LTE bandwidth } & $20 \mathrm{MHz}$ \\
\hline \multicolumn{3}{|c|}{ LTE transport protocol } & UDP \\
\hline \multicolumn{3}{|l|}{ Wi-Fi bandwidth } & $20 \mathrm{MHz}$ \\
\hline \multicolumn{3}{|l|}{ Wi-Fi standard } & IEEE $802.11 \mathrm{n}$ \\
\hline \multicolumn{3}{|c|}{ Wi-Fi transport protocol } & UDP* \\
\hline \multicolumn{3}{|l|}{ Path loss model } & $\begin{array}{l}\text { IEEE } 802.11 \text { ax Indoor } \\
\text { Propagation Loss Model }\end{array}$ \\
\hline \multirow[t]{8}{*}{ Transmit Power } & BS & TxPower & $18 \mathrm{dBm}$ \\
\hline & & TxGain & $5 \mathrm{~dB}$ \\
\hline & & RxGain & $5 \mathrm{~dB}$ \\
\hline & & NoiseFigure & $5 \mathrm{~dB}$ \\
\hline & UE & TxPower & $18 \mathrm{dBm}$ \\
\hline & & TxGain & $0 \mathrm{~dB}$ \\
\hline & & RxGain & $0 \mathrm{~dB}$ \\
\hline & & NoiseFigure & $9 \mathrm{~dB}$ \\
\hline \multicolumn{3}{|l|}{ Mobility } & None \\
\hline \multicolumn{3}{|c|}{ Sensing thresholds for LTE and Wi-Fi } & $-62.0 \mathrm{dBm}$ \\
\hline \multicolumn{3}{|l|}{ MIMO } & None \\
\hline
\end{tabular}

*By applying TCP instead of UDP, we can observe small throughput degradation over both of LTE and Wi-Fi

center position. The Wi-Fi stations (STAs) and LTE user equipment (UE) are circularly located at a distance of $10 \mathrm{~m}$ from the center. The 8UEs/8STAs and 12UEs/12STAs are tested for the low-interference environment. A more complicated environment, namely a high-interference environment, is tested in Figure $6 \mathrm{~b}$. The center co-location of 1 eNodeB and $1 \mathrm{AP}$ is identical to that in the low interference environment. However, additional Wi-Fi APs are installed to ensure additional interference. Furthermore, LTE UEs Wi-Fi STAs are closely located to maximize their interference. Moreover, 8UEs/8STAs and 12UEs/12STAs are also tested. Table 1 shows other system model for the simulations.

In addition, we show the modification of NS-3 for simulations. Table 2 shows NS-3 modifications.

\subsection{Simulations}

To define the parameters of function (6) for each interference environment, we use a small number of training data obtained from simulations. The Table 3 in the

Table 2 Modified NS-3 modules

\begin{tabular}{ll}
\hline Module name & Modified contents \\
\hline laa-wifi-simple.cc & Parameter setup \\
scenario-helper.cc & Scenario setup \\
laa-wifi-coexistence-helper.cc & Traffic setup, LBT operation
\end{tabular}


Appendix shows the extensive simulation results for every dual threshold $(i T H, d T H)$. The only small selected simulations are sufficient to define the function (6). Approximately $8 \sim 10$ simulations for each interference environment are applied to define the parameters of function (6). This applicability gives a strong advantage to define function (6) for various inference environments those are not exploited in our test experiments.

After the defining the function (6) for test environments, we apply the dual threshold update procedure presented in Section 3. With respect to each test environment, the operation commences with a randomly selected dual threshold, and the aforementioned threshold update procedure is then applied. Figure 7 shows the effectiveness of the procedure. The proper dual threshold could be determined for each weight within a very small time bound. The highest converging time of the dual threshold corresponds to within 2000 $\mathrm{ms}$, and the maximum number of procedure steps corresponds to less than 18. The $x$-axis in Fig. 7 denotes the obtained $\{i T H, d T H\}$ pairs and their weights.

Now, we demonstrate that the obtained dual threshold is close to the optimal value. Numerous search graphs are illustrated for test environments to identify the optimality. Figure 8 illustrates the enumerative space for dual threshold pairs. The $x$-axis and $y$-axis denote $i T H$ and $d T H$, respectively. The lowest plane corresponds to the highest weight for function $f(i T H$, $d T H$ ) (i.e., assigned weight is 1.0), and conversely, the highest plane corresponds to the lowest weight.

Balanced points can be determined from the 3D performance illustration. Both LTE and Wi-Fi could only obtain balanced throughputs under similar values of $i T H$ and $d T H$. Specific pairs of $i T H$ and $d T H$ obtain a good performance in all the test cases. The pairs of $(i T H, d T H)=\{(3,3),(4,4),(7,7),(7,8)\}$ indicate a good balance of LTE and Wi-Fi. The dual threshold pair $(7,7)$ shows the smallest throughput difference between LTE and Wi-Fi in the "Low Interference: 12UEs/12STAs" case. The same dual threshold is obtained in Fig. 7a in which the value is obtained by the proposed threshold update procedure as described in Section 3. The proper $i T H$ and $d T H$ as the similar values (i.e., $(i T H, d T H)=(3,3),(4,4),(7,7),(7,8))$ denotes that the repetitive extension of ECCA sensing

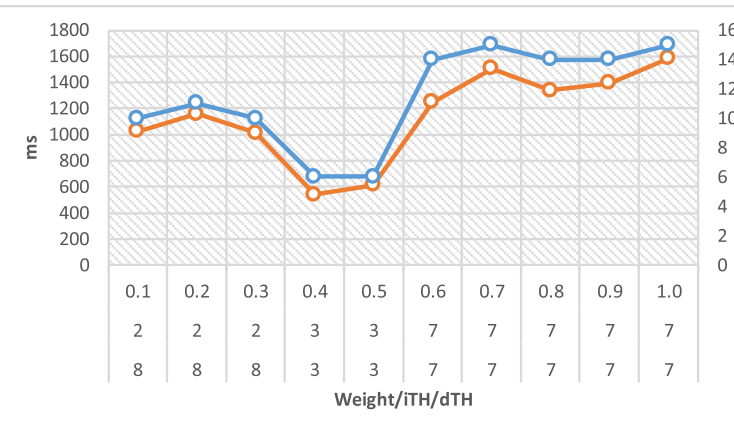

- Converging Time (ms)

$\multimap$ \#of Steps to Converge

(a) Low Interference: 12UEs/12STAs

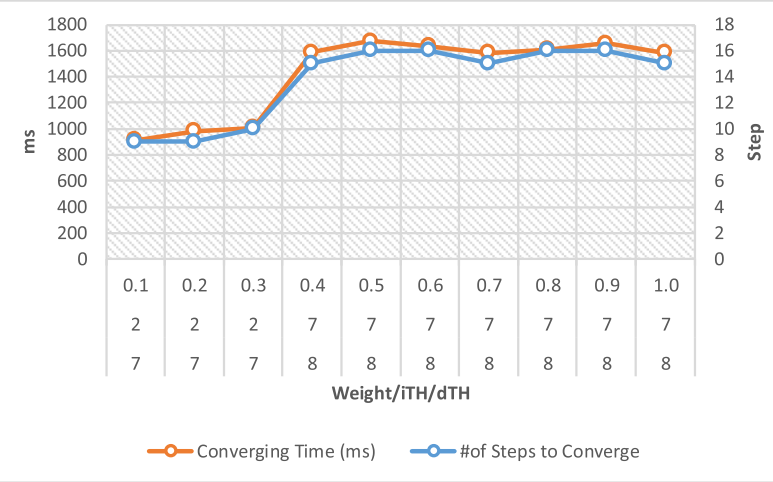

(c) Low Interference: 8UEs/8STAs

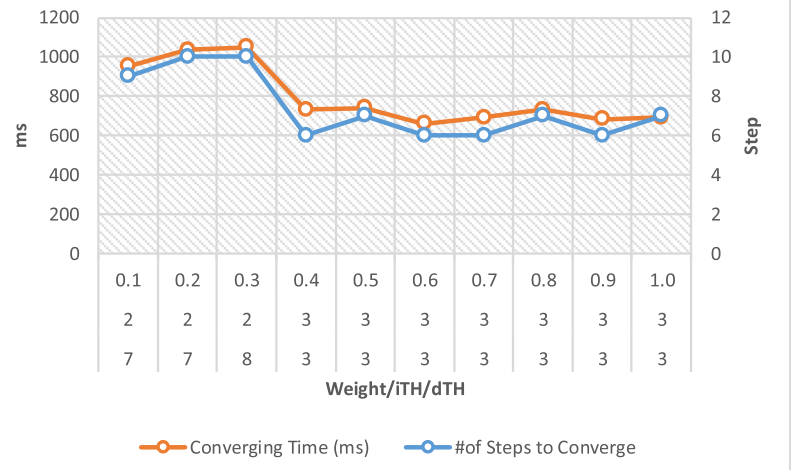

(b)High Interference: 12UEs/12STAs

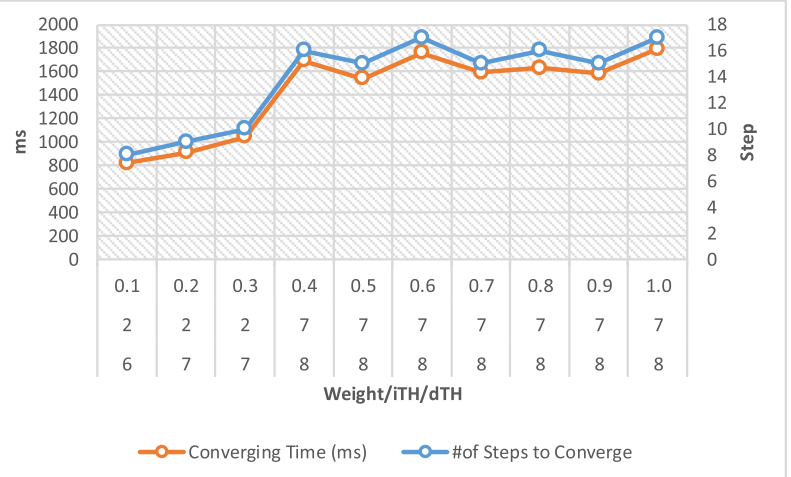

(d) High Interference: 8UEs/8STAs

Fig. 7 Convergence of thresholds. a Low interference: 12UEs/12STAs. b High interference: 12UEs/12STAs. c Low interference: 8UEs/8STAs. d High interference: 8UEs/8STAs 


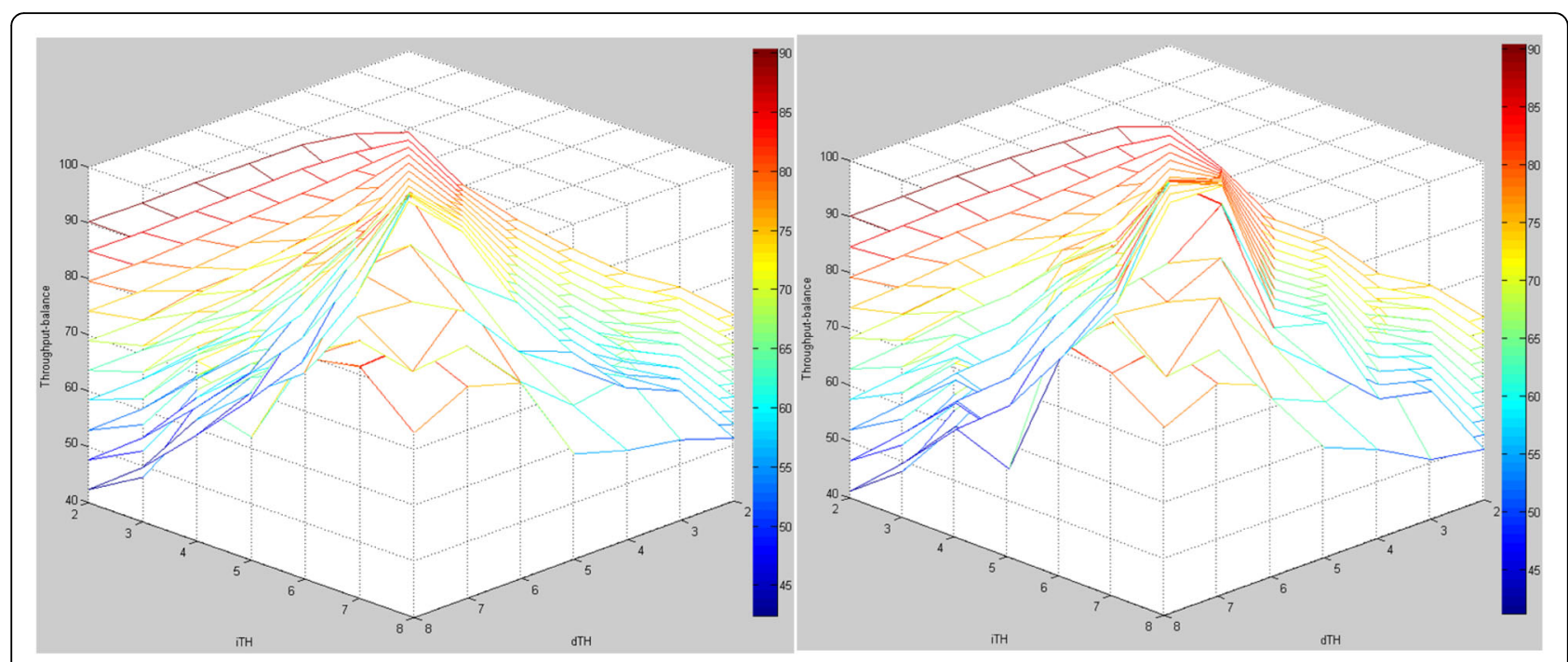

(a) Low Interference: 12UEs/12STAs

(b) High Interference: 12UEs/12STAs

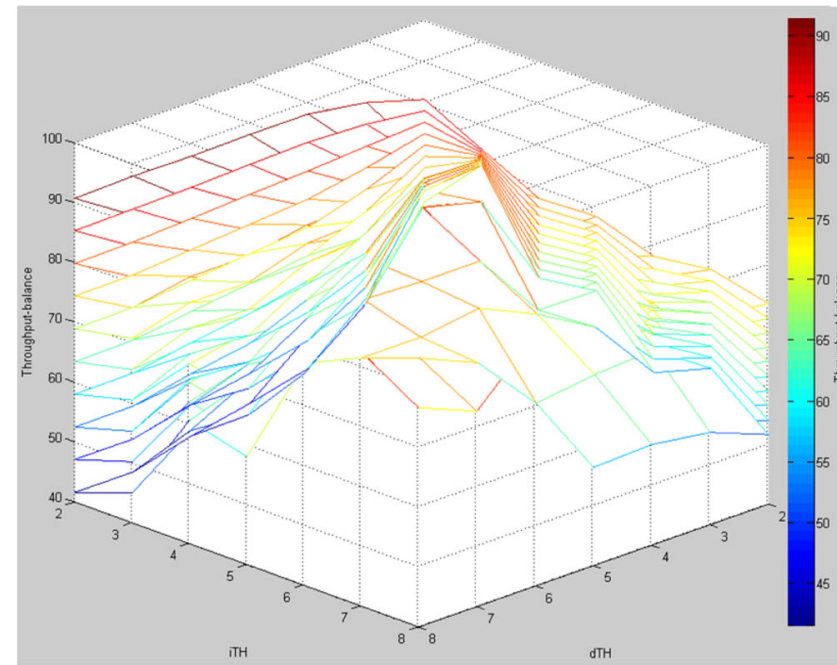

(c) Low Interference: 8UEs/8STAs

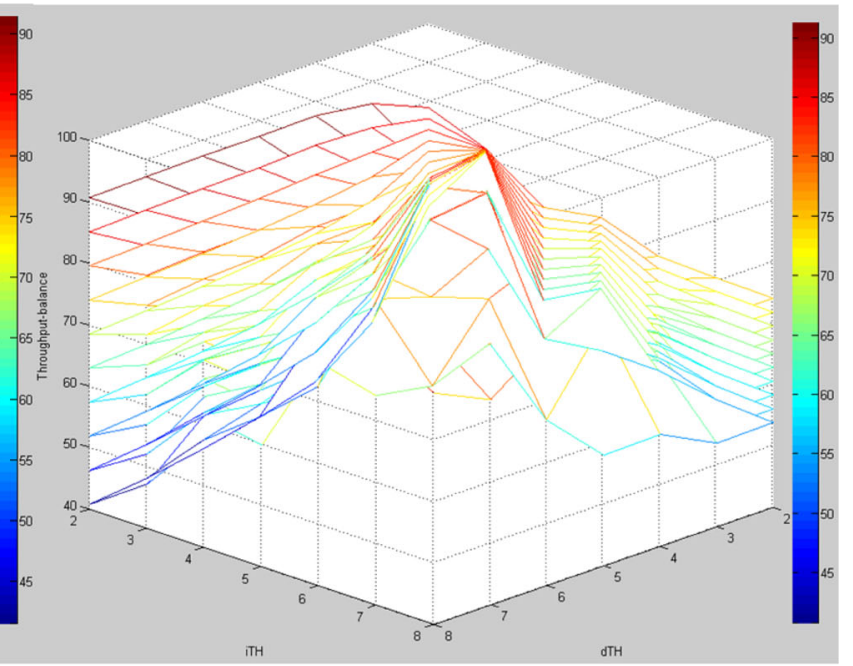

(d) High Interference: 8UEs/8STAs

Fig. 8 3D Performance graph. a Low interference: 12UEs/12STAs. b High interference: 12UEs/12STAs. c Low interference: 8UEs/8STAs. d High interference: 8UEs/8STAs

time is not appropriate to achieve good performance. In traditional random backoff mechanism, the sensing time is exponentially extended by the repetitive channel access failure. However, the $i T H$ and $d T H$ as the similar values prevent the repetitive extension of sensing time. Within a small number of access failures after the extension of ECCA sensing time, the maximum ECCA sensing time resets to the minimum value by the action of $d T H$ (the $d T H$ has a similar value of $i T H)$. Remember the example of Action: $i T H$ $=d T H=4$ in Fig. 4. The same value of $i T H$ and $d T H$ restricts the repetitive extension of sensing time to only a single time.
Figure 9 shows the performance comparison between the proposed dual threshold control method and the well-established random backoff algorithm. The random backoff algorithm is applied to both the $\mathrm{LBE}$ and FBE with variable size CW. It should be noted that the difference between LBE and FBE is not significant. The results indicated that the advantage of the proposed method improves in the case of a high-interference environment. The high interference: 12UEs/12STAs case shows a maximum enhancement of $7.6 \%$ for the weight 1.0 (the average enhancement for every weight corresponds to $6.6 \%$ ). The other cases show enhancement in the 


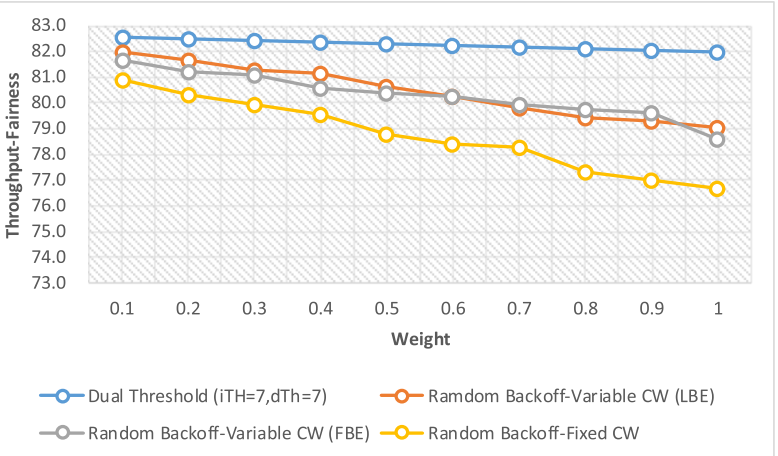

(a) Low Interference: 12UEs/12STAs

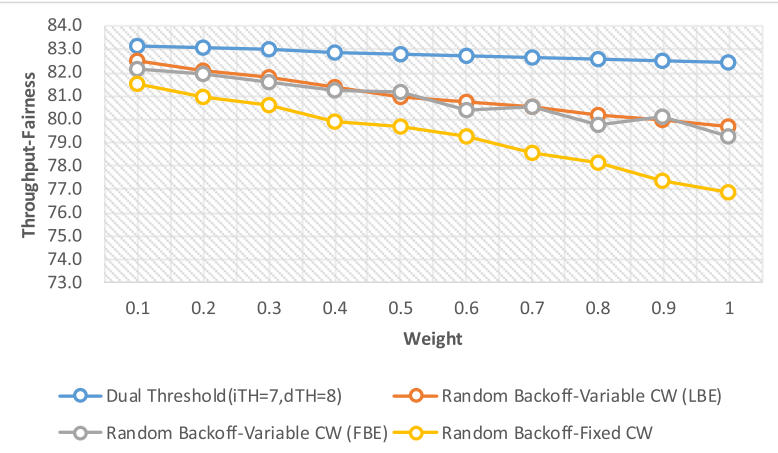

(c) Low Interference: 8UEs/8STAs

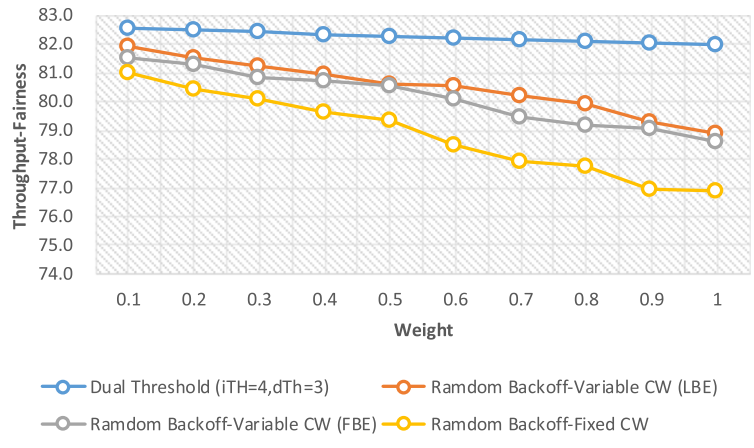

(b) High Interference: 12UEs/12STAs

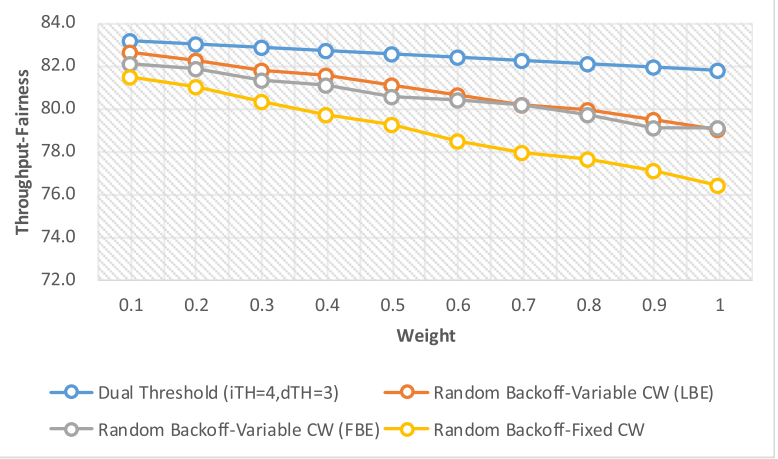

(d) High Interference: $8 \mathrm{UEs} / 8 \mathrm{STAs}$

Fig. 9 Performance comparison. a Low interference: 12UEs/12STAs. b High interference: 12UEs/12STAs. c Low interference: 8UEs/8STAs. d High interference: 8UEs/8STAs

approximate range of $4.3-7.2 \%$. Note that, we add the results of random backoff with fixed size $\mathrm{CW}$. The performance of fixed size $\mathrm{CW}$ is not considerable for comparison.

Figures 10 and 11 present separate figures of the throughput and the fairness metric. We can observe that the fairness metric is the more dominant factor for performance enhancement of the proposed dual threshold access control method.

\section{Concluding remarks}

It is necessary for wireless service operators to build the best service portfolio strategy for each user by focusing on the maximum benefit for network convergence while

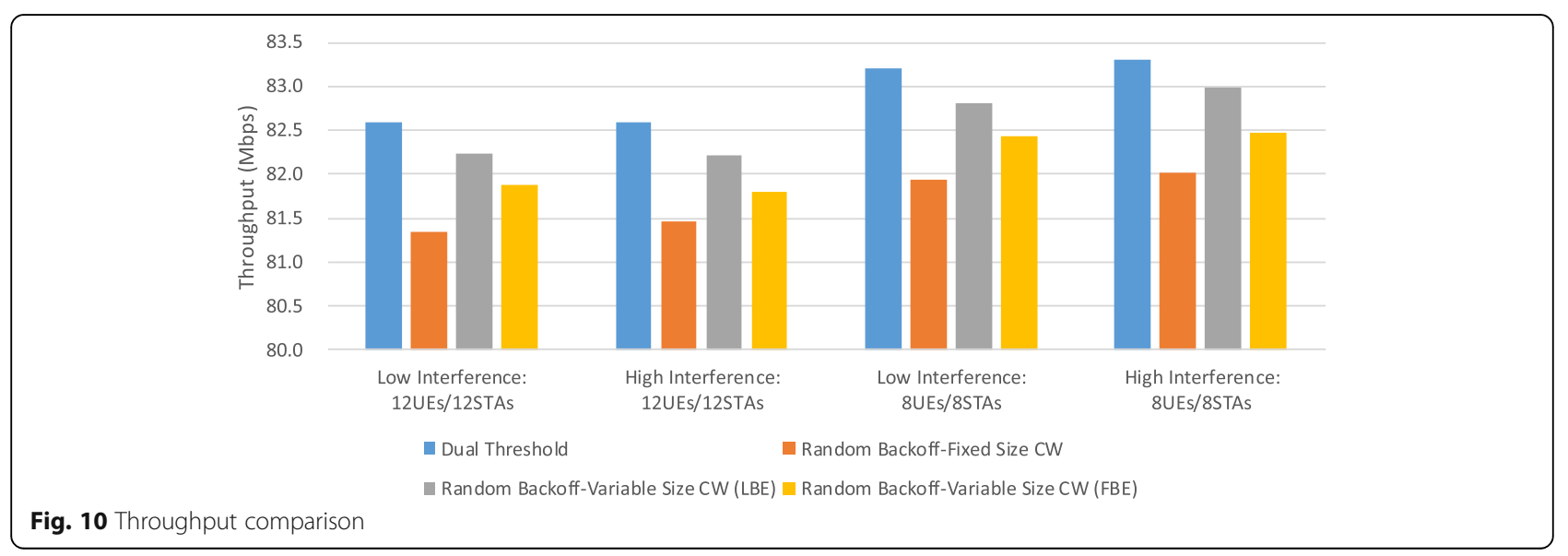




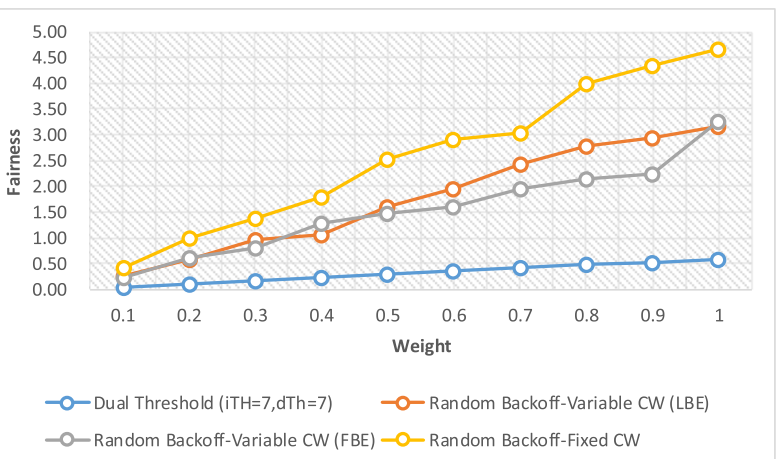

(a) Low Interference: 12UEs/12STAs

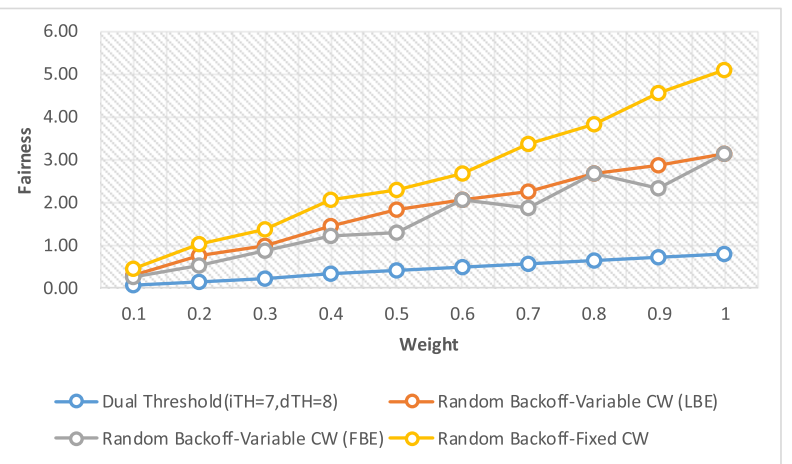

(c) Low Interference: 8UEs/8STAs

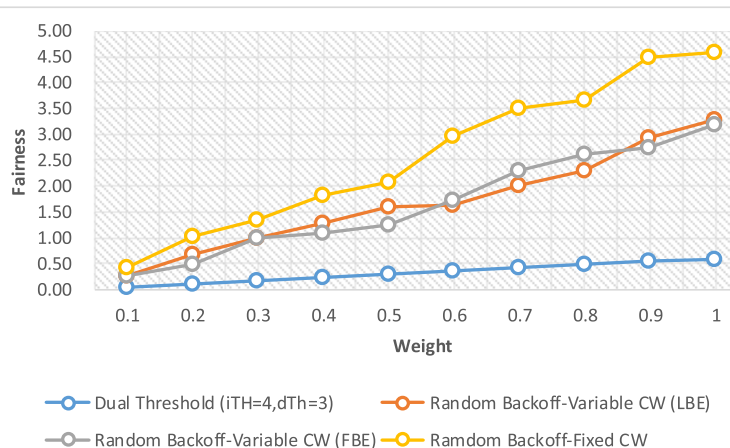

(b) High Interference: 12UEs/12STAs

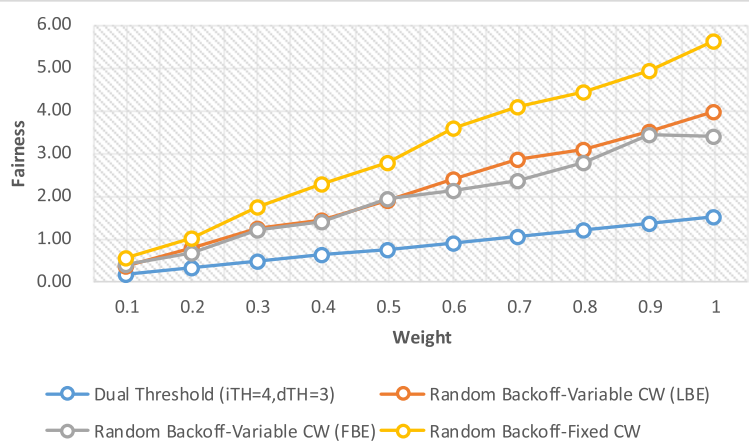

(d) High Interference: $8 \mathrm{UEs} / 8 \mathrm{STAs}$

Fig. 11 Fairness comparison. a Low interference: 12UEs/12STAs. b High interference: 12UEs/12STAs. c Low interference: 8UEs/8STAs. d High interference: 8UEs/8STAs

maximizing the overall network utilization. The LTELAA is one of the best methods to obtain a connection set for every user in multiple access networks. The proposed channel access method improves the balance between LTE and Wi-Fi for the LTE-LAA. The number of consecutive ECCAs is counted, and the dual threshold is applied to obtain a proper CW size. The dual threshold mechanism guarantees sufficient opportunities to access shared channels to the LTE-LAA while maintaining fair resource sharing between coexisting Wi-Fi users.

The study involved developing a fast and efficient converging scheme for a dual threshold. A gradient climbing method was adopted to update the dual threshold. The extensive enumerative simulations demonstrate the near optimality of the proposed approach. The overall decision process is unique and useful for unlicensed bands. The utility of the proposed dual threshold and the updating method in an LTE-LAA operation framework provides opportunities to design fair sharing for unlicensed resources. The network effect of a newly designed access scheme can be tested and adapted to any given network environment even under very serious interferences. The simple and practical design scheme demonstrated in the present study provides an appropriate decision-making process for new LTE-LAA adaptation in a network service.

\section{Appendix}

The results of the four scenarios are listed in Table 3. The range of $i T H$ and $d T H$ is set as $2-8$ for the simulation in the study. Table 3 shows simulated results of proposed dual thresholds. The figures in the Table 3 are the throughput of LTE and Wi-Fi (per network). Each throughput value is calculated from the $(i T H, d T H)$. Figure5 shows the holistic throughput pattern. We can roughly find the balance points of total throughout and fairness in the Fig. 5. However, the generic form of gradient descent method needs detailed throughput data of LTE and Wi-Fi. The data of Table 3 can be useful input of the generic form of gradient descent method. 


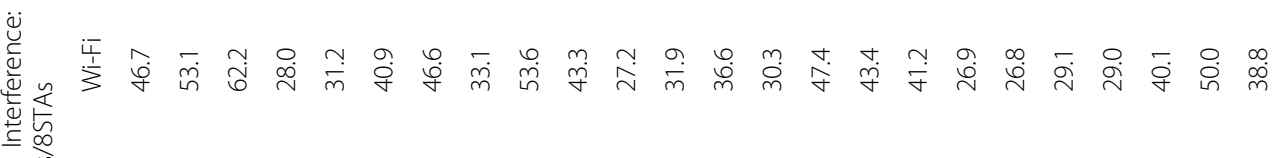

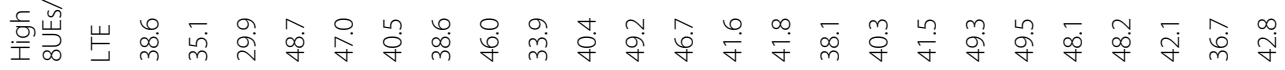

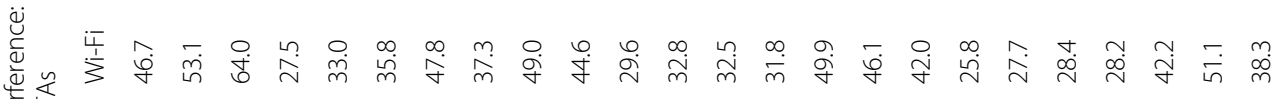

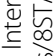

产岀

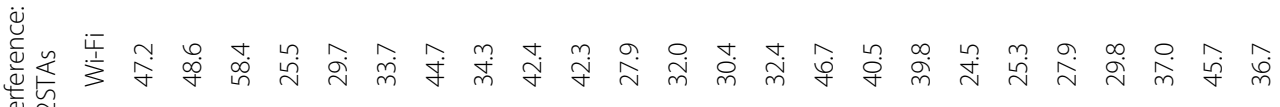
离

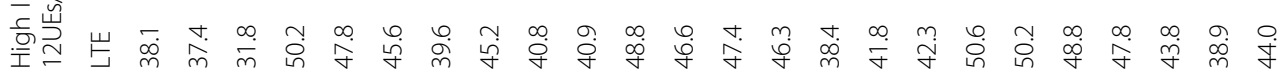

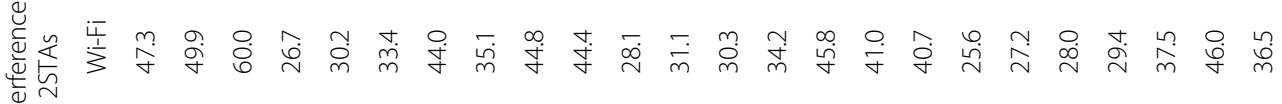

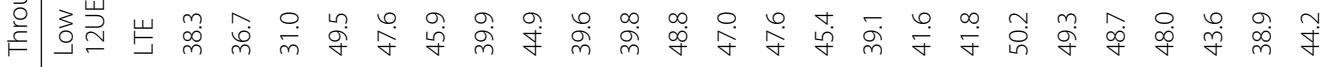

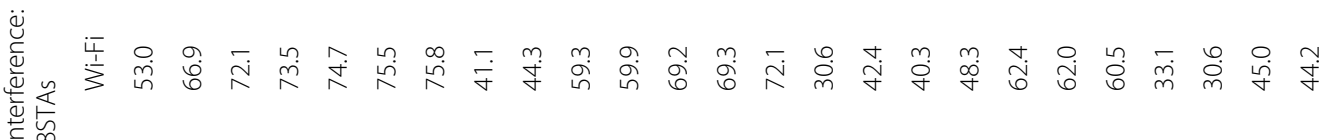

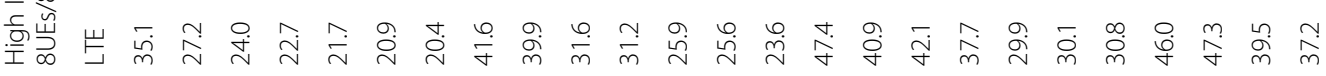

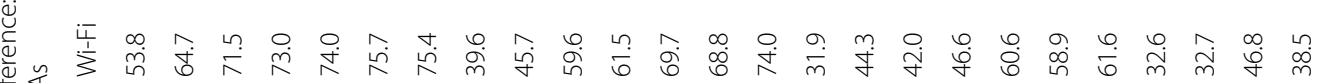

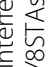

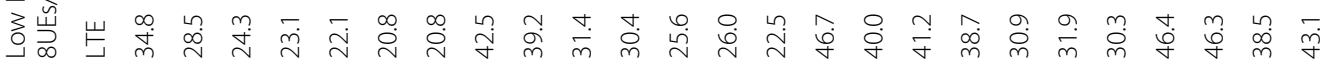

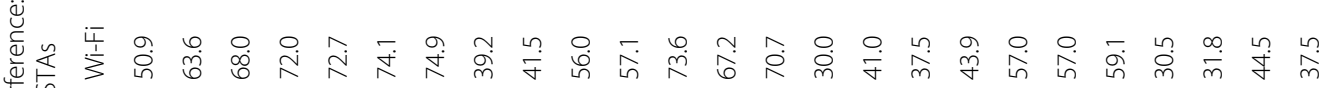

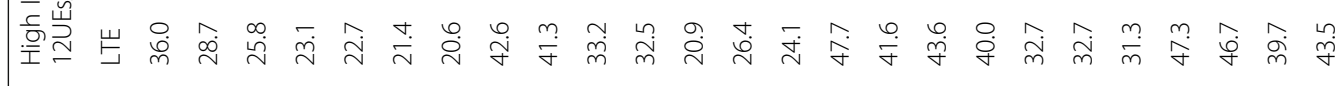

a)

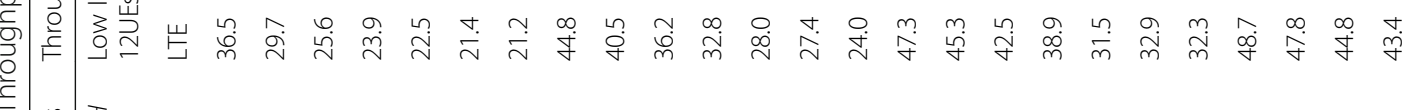




\section{Authors' contributions}

JHK is the first author of the manuscript. He developed algorithmic processes and mathematical interpretations. In addition, he designed the simulator and experiments. SC is the second author of the manuscript. He built the simulator and perform all experiments. He also suggested the framework of dual threshold control mechanism. All authors read and approved the final manuscript.

\section{Funding}

This work was supported by the Institute for Information \& communications Technology Promotion (IITP) grant funded by the Korea government (MSIP) (No.2016-0-00160, Versatile Network System Architecture for MultiDimensional Diversity, No.2016-0-00151, Development of LTE Small Cell supporting LWA/ LAA dual mode). This work is supported by the National Research Foundation of Korea (NRF) grant funded by the Korea government (MSIT) (No. NRF-2017R1A2B1009709)

\section{Competing interests}

The authors declare that they have no competing interests.

\section{Author details}

${ }^{1}$ Department of Industrial Engineering, Ajou University, 206, World cup-ro, Yeongtong-gu, Suwon-si, Gyeonggi-do 16499, Korea. ${ }^{2}$ Division of Device Solution, Samsung Electronics, Seoul, South Korea.

Received: 31 January 2018 Accepted: 24 June 2019

Published online: 16 July 2019

\section{References}

1. Cisco Visual Networking Index: global mobile data traffic forecast Update, 2014-2019, 1st ed. Cisco Public, 2015, pp. 3-16.

2. EverythingRF, https://www.everythingrf.com/community/what-is-lte-licenseassisted-access.

3. E. H. Ong, J. Kneckt, O. Alanen, Z. Chang, T. Huovinen, Timo Nihtilä , "IEEE 802.11 ac: Enhancements for very high throughput WLANs", PIMRC 2011

4. S. Dimatteo, P. Hui, B. Han, V.O.K. Li, in 8th IEEE International Conferece on Mobile Ad-Hoc Sensor Systems. Cellular traffic offloading through WiFi networks (2011)

5. J. Jeon, Q. Li, H. Niu, A. Papathanassiou, G. Wu, LTE in the unlicensed spectrum: a novel coexistence analysis with WLAN systems. Globecom (2014)

6. F. Liu, E. Bala, E. Erkip, R. Yang, in 2011 International Symposium on Modeling and Optimization in Mobile, Ad Hoc and Wireless Networks (WiOpt). A framework for femtocells to access both licensed and unlisenced bands (2011)

7. A.M. Cavalcante, E. Almeida, R.D. Vieira, S. Choudhury, E. Tuomaala, K. Doppler, Performance evaluation of LTE and WiFi coexistence in unlicensed bands. 2013 IEEE 77 th Vehicular Technology Conference (VTC Spring) (2013)

8. E. Almeida, A.M. Cavalcante, R.C.D. Paiva, F.S. Chaves, F.M. Abinader Jr., R.D. Vieira, Enabling LTEMiFi coexistence by LTE blank subframe allocation. ICC (2013)

9. Qualcomm, "LTE-U technology and coexistence", LTE-U Forum, 2015

10. Ericsson, "LTE-U coexistence", LTE-U Forum, 2015

11. R. Ratasuk et al., "License-exempt LTE deployment in heterogeneous network," in Proc. IEEE Int. Symp. Wireless Commun. Syst. (ISWCS), Paris, France, Aug. 2012, pp. 246?250

12. R. Ratasuk, N. Mangalvedhe, and A. Ghosh, "LTE in unlicensed spectrum using licensed-assisted access," in Proc. IEEE GLOBECOM,Austin, TX, USA Dec. 2014, pp. 746?751

13. C. Chen, R. Ratasuk, A. Ghosh, "Downlink performance analysis of LTE and Wi-Fi coexistence in unlicensed bands with a simple Listen-Before-Talk scheme," IEEE 81st. Vehicular Technology Conference (VTC Spring), 2015.

14. ETSI EN 301 893, Harmonized European Standard, "Broadband radio access networks (BRAN); 5 GHz high performance RLAN(v1.7.2)," July. 2014.

15. J.S. Um, S.J. Yoo, S.K. Park, A study on channel access mechanism of LTE for coexistence with Wi-Fi on $5 \mathrm{GHz}$ unlicensed spectrum. The Journal of Korean Institute of Electromagnetic Engineering and Science (2015)

16. B. Chen, J. hen, Y. Gao and J. Zhang, "Coexistence of LTE-LAA and Wi-Fi 5GHz with corresponding development scenarios: a survey", IEEE Communications Survey and Tutorials, Vol.19, No.1, 2017

17. Monica Paolini, "LTE unlicensed and Wi-Fi: Moving beyond coexistence,"Senza Fili, Sammamish, WA, USA, Tech. Rep., 2015. [Online]. Available: https://www.slideshare.net/SmallCellForum1/Ite-unlicensed-andwifi-moving-beyond-coexistence-from-monica-paolini-senza-fili
18. 3GPP,"3rd generation partnership project; feasibility study on licensedassisted access to unlicensed spectrum; study on licensed-assisted access to unlicensed spectrum (release 13)," 3GPP, Sophia Antipoles, France, TR 36.889 V13.0.0, Jul. 2015.

19. Rui Yin, Guanding Yu, Amine Maaref, and Geoffrey Ye Li, " Adaptive LBT for licensed assisted access LTE networks ", IEEE Globecom 2015

20. Tao Tao, Feng Han and Yong Liu "Enhanced LBT algorithm for LTE-LAA in unlicensed band", IEEE PIMRC 2015

21. F. Hao, C. Yongyu, H. Li, J. Zhang, W. Quan, Contention window size adaptation algorithm for LAA-LTE in unlicensed band. ISWCS (2016)

22. Anand M. Baswade and Bheemarjuna Reddy Tamma, "Channel sensing based dynamic adjustment of contention window in LAA-LTE networks", COMSNETS 2016

23. Salman Saadat, Da Chen, Kai Luo, Mingjie Feng, and Tao Jiang, " License assisted access-WiFi coexistence with TXOP backoff for LTE in unlicensed band", China Communications, Vol.14, Issue 3, 2017

24. 3GPP TS 37.213, "Physical layer procedures for shared spectrum channel access (Release15), 2018.9

25. Chris Burges, Tal Shaked, Erin Renshaw, Ari Lazier, Matt Deeds, Nicole Hamilton, Greg Hullender, "Learning to rank using gradient descent", ICML '05 Proceedings of the 22nd international conference on Machine learning, 2005, Pages $89-96$

\section{Publisher's Note}

Springer Nature remains neutral with regard to jurisdictional claims in published maps and institutional affiliations.

\section{Submit your manuscript to a SpringerOpen ${ }^{\circ}$ journal and benefit from:}

- Convenient online submission

- Rigorous peer review

- Open access: articles freely available online

High visibility within the field

- Retaining the copyright to your article

Submit your next manuscript at $>$ springeropen.com 